\title{
Optimal Pricing Strategy for Residential Electricity Usage in Smart Grid
}

\author{
Quan-Hui Liu*, Yingjie Zhou ${ }^{\dagger}$, Zhongtao Yue*, Bidushi Barua ${ }^{\ddagger}$, and Yanru Zhang* \\ ${ }^{*}$ CompleX Lab, School of Computer Science and Engineering, University of Electronic Science and \\ Technology of China, Chengdu, Sichuan, China, 611731 \\ ${ }^{\dagger}$ College of Computer Science, Sichuan University, Chengdu, Sichuan, China, 610065 \\ $\ddagger$ Centre for Wireless Communications, University of Oulu, Oulu, Finland, 90014
}

\begin{abstract}
Electricity Retailers offer various utility plans in the hope that the increased competition would result in lower prices, improved service, and innovative product offerings. In this paper, we present the retail electric provider's (REP) optimal pricing strategy for residential customers in smart grid, in which the REP offers multiple utility plans for customers with different needs, which includes a flat-rate plan, a multistage plan, and a lump-sum fee plan. The residential customers select the utility plan that maximize their own payoffs by considering their own demands and the pricing strategies of the three plans. In the other way around, the REP optimizes its profit by carefully designing its pricing strategy based on residential customers' decisions. To obtain insights of such a highly coupled system, we consider a system with one REP and a group of customers in need of electricity. We propose a three-stage Stackelberg game model, in which the REP acts as the leader who decides the specific plans to offer at Stage I, then announces the price for each plan in stage II, and finally the customers act as followers that select plans in stage III. We derive the market equilibrium by analyzing customers' decisions among the plans under different pricing schemes. Then, we provide the REP's optimal pricing strategies to maximize its profit. In the end, we give the optimal decisions for REP on the specific plan(s) to offer while considering each customer's evaluation and demand. Both the analytical and simulation results show that the lump-sum fee plan can maximize REP's profit in most cases.
\end{abstract}

\section{INTRODUCTION}

Over the past several decades, many US states have moved to deregulate their electric markets, with 24 states allowing for at least some competition among retail electric providers (REPs) including California, Texas, and New York [1]. Deregulation on the retail market has enabled all electricity customers, both business entities and residential customers to choose not only their REP among various retailers who provide a range of options such as lower price, better service, eco-friendliness and so on, but also the plan that appeals to them based on price, term, or another factors [2]. Texas has the most competitive retail electricity market in the United States. As of September 2016, 109 REPs were operating in the Electric Reliability Council of Texas and 97 products with $100 \%$ renewable energy were available to consumers [3]. Texas's competitive electricity market results in a noticeable retail price decline in recent years.

As electric vehicle and other power applications emerge, residential electricity demand is continuously increasing in

The work of Yingjie Zhou is partly supported by National Natural Science Foundation of China (NSFC) with grant number 61801315.

All corresponding to Yanru Zhang (yanruzhang@uestc.edu.cn) and Yingjie Zhou (yjzhou@scu.edu.cn). recent years [4]. According to a report by the Edison Electric Institute (EEI) and the Institute for Electric Innovation (IEI), the residential electric vehicle charging demand in U.S. will be doubled from 2018 in less than 3 years [5]. To satisfy the increasing residential electricity demand and provide high quality service in the future, demand side management (DSM) has been proposed for smart grid in response of the residential electricity demand. For example, altering customers' electricity consumption behaviors by shifting electricity usage from peak to non-peak hours through load scheduling or pricing strategies [6]. The real price impact of retail electricity choice is difficult to measure because of, among other things, rate variations with respect to wholesale price, the customer's load profile, on- and offpeak conditions, marketing costs, and contract duration [7]. Both the technology and economics aspects of residential electricity demand response have attracted much attention of the industry and academia [8].

To increase market shares and profits, the REPs are actively designing various electricity plans to attract potential consumers [9]. One traditional way to charge customer is the flat rate pricing, which offers a fixed rate and charge customers based on their electricity usage [10]. Another electricity plan is the multi-stage electricity price (MEP) policy [11]. This plan is widely adopted in many countries, including plenty of areas in China [12]. Time-of-use (TOU) pricing is another plan that charges the customers for different prices in on-peak hours and off-peak hours. It can guide the customers to regulate electricity consumption in terms of fairness and efficiency [13], and has been approved to be able to reduce peak power consumption [14]. From the customers' point of view, shopping deregulated electricity plans can be overwhelming, since they find out the most suitable plan from their subscribed REP depending upon many factors such as their needs and/or interests, and the pricing strategies of REP.

In this paper, we present REP's optimal pricing strategy for residential electricity usage that supports different subscription options. We model the operation of multiple plans as a three-stage Stackelberg game to derive REP's optimal pricing strategy and decisions. On one hand, the REP, which acts as a leader, needs to decide on its pricing strategy to optimize its profit while ensuring residential customers' participation. On the other hand, the residential customers, which act as followers, need to decide on their subscription strategies to optimize a tradeoff between the benefit from 
electricity plans and the associated payment cost.

The main contributions of this paper are summarized as follows:

- System model with multiple electricity plans for residential customers: We introduce the optimal charging strategy that considers different electricity plans and relevant pricing strategy for residential electricity usage in smart grid. We model the research problem as a threestage Stackelberg game and derive the optimal pricing strategy and decisions through backward induction.

- Analysis of parameters' influences: We study the impacts of the customers characteristics and electricity cost on the REP's profit and market state. Our results provide the REP with guidelines for selecting the specific plan(s) to maximize profit.

- Comparison between plans: We compare the traditional flat-rate plan, multi-stage plan, and lump-sum fee plan. Our results provide insights for REPs to make optimal pricing strategy to maximize profit.

The key insights of this paper are summarized as follows:

- Lump-Sum Fee Plan maximizes profit in most cases. In most cases, especially when customers have high evaluation and consumes large electricity, lump-sum fee plan brings the highest profit to REP than other plans.

- Serving flat-rate plan only when customers' evaluation and demand is medium. When customers have lower evaluation and demand less electricity, they can not afford high price, nor consume large amount of electricity. In this case, customers prefer the flat-rate plan most, REP should offer customers flat-rate plan to maximize its profit.

- Customer Decisions: Customers with high evaluation and demand prefer lump-sum fee plan, while customers who have low evaluation and demand prefer the traditional flat-rate plan. Customers who feel the price is too high will not choose any plan.

- Key Parameters: REP's profit increases with customers' evaluation and demand, but decreases with electricity cost. Furthermore, large electricity cost lowers the fraction of customers choosing lump-sum fee plan, while large customer evaluation increases the fraction of customers choosing lump-sum fee plan.

The rest of this paper is organized as follows. First, we introduce the system model and three electricity plans in Section II. Then, the three-stage Stackelberg game is solved in Section III, in which we use backward induction to derive customers' optimal decisions, REP's optimal pricing strategy, and optimal decision sequentially. The numerical results are provided in Section IV. Finally, conclusions are drawn in Section V.

\section{SySTEM MOdEL}

Considering monopoly market with only one electricity retailer, the REP, who supplies electricity to a group of residential customers. The REP offers different electricity plans and charges its customers with the corresponding pricing strategy. We model the interactions of a REP and its customers as a three-stage Stackelberg game. Specifically, the REP decides which plans will be provided in Stage I, including the flat-rate plan, multi-stage plan, and lump-sum fee plan. In Stage II, the REP computes the optimal prices of each service plan to maximize its expected profit from the customers. In Stage III, each customer decides which plans to take based on its expected payoffs under the announced prices.

\section{A. Modeling Preliminaries}

1) Pricing Strategy: The 3 electricity plans flat-rate plan, multi-stage plan, and lump-sum fee plan are denoted as $r_{1}, r_{2}$ and $r_{3}$, respectively. Each plan has a specific pricing strategy to charge customers the amount of electricity they have consumed $q$. In service plan $r_{1}$, the REP charges customers by a flat unit fee $p$, the unit electricity fee is fixed no matter how much the customers have consumed, and the total fees is increasing with the electricity consumed linearly. Compared with service plan $r_{1}$, we propose a two-stage plan which is a special case of multi-stage plan when there are 2 different rates in service plan $r_{2}$. When the electricity consumed is less than or equal to a threshold $q_{t}$, the unit price is $\alpha p$. When the total usage of electricity is larger than $q_{t}$, the overage unit price becomes $\beta p$. In such a way, the REP can encourage the use of electricity by setting $\alpha>\beta$ if the electricity is over capacity. However, by setting $\alpha<\beta$, the REP discourages the customers of consuming more electricity. For service plan $r_{3}$, customers are usually charged a monthly service fee and additional charges based on the electrical energy consumed by the household during the month. The REP charges a lump-sum fee $\gamma$ whenever customer consumes less than $q_{t}$, then charges the overage part with a unit price $\beta^{\prime} p$. We assume that $\beta^{\prime}=\beta$. When customer's electricity usage has surpassed $q_{t}$, the prices for overage are the same in both Plan 2 and Plan 3.

In this work, we focus on the REP's optimal pricing policy under different service plans, i.e., $r_{1}, r_{2}$ and $r_{3}$. Since the traditional way flat rate $p$ has a commonly accepted value, we assume $p$ in Plan 1 is a constant value. We are concentrating on solving the optimal prices in Plan 2 and 3, which are defined as follows: $\left\{r_{2}\right\} \Rightarrow\left\{\alpha^{\star}\right\}$, and $\left\{r_{3}\right\} \Rightarrow \gamma^{\star}$. Then we show the REP's optimal decisions between the traditional pricing and our proposed ones.

2) Customer Characteristics: Assuming that customer's utility function is modelled by a concave function of its electricity consumption $v(q)$, which is increasing with the electricity consumption $q$. Customer's electricity consumption $q$ is modelled as a random variable and follows a uniform distribution over the range $[0, \bar{q}]$, where $\bar{q}$ is the maximum electricity consumption. For the sake of simplicity, we adopt the special form of a concave function, and write the utility function $v(q)$ in the linear form as $v q$, where $v$ represents the customer evaluation coefficient for a unit amount of electricity. User's eva;uation can be regard as user's affordability, which is related with their income status and life style. Similar to the customer's consumption, we assume that customer's evaluation follows the uniform distribution over the range $[0, \bar{v}]$, where $\bar{v}$ is the maximum evaluation coefficient. 


\section{B. Customer Payoff}

Generally, different customers have different electricity demand and their evaluations for the same electricity consumption vary from one to another. Thus, we use $(v, q)$ to characterize one customer's evaluation and electricity demand. For each service plan the REP provided, the payoff of a type- $(v, q)$ customer depends on both customer's evaluation coefficient $v$ and his/her electricity demand $q$. Customer's payoff by subscribing to different electricity plans are listed as follows.

1) Plan 1: For flat-rate plan, i.e., $r_{1}$, the payoff for a type- $(v, q)$ customer is

$$
S_{1}=v q-q p .
$$

For the type- $(v, q)$ customer, its electricity consumption $q$ is unknown prior to it subscription. Based on our previous assumption that the consumption follows the uniform distribution in the range $[0, \bar{q}]$ with the probability density function (PDF) $f(q)=\frac{1}{\bar{q}}$. Thus, for customer with the type- $(v, q)$ choosing $r_{1}$ plan, its expected payoff is as follows:

$$
\overline{S_{1}}=\int_{0}^{\bar{q}} S_{1} f(q) d q .
$$

Thus, for a flat-rate plan with a fixed electricity price, the higher the customer's evaluation $v$ and the more electricity it consumes, the higher income would bring to the REP.

2) Plan 2: When a type- $(v, q)$ customer chooses the twostage plan $r_{2}$, his/her payoff is:

$$
S_{2}= \begin{cases}v q-\alpha p q, & q \leq q_{t}, \\ v q-\alpha p q_{t}-\beta p\left(q-q_{t}\right), & q>q_{t} .\end{cases}
$$

In the same way, we can compute the expected payoff of a customer who subscribed to the $r_{2}$ plan with type- $(v, q)$ as follows:

$$
\begin{aligned}
\overline{S_{2}} & =\int_{0}^{\bar{q}} S_{2} f(q) d q \\
& =\int_{0}^{q_{t}}[v q-\alpha p q] f(q) d q \\
& +\int_{q_{t}}^{\bar{q}}\left[v q-\alpha p q_{t}-\beta p\left(q-q_{t}\right)\right] f(q) d q .
\end{aligned}
$$

3) Plan 3: For the customers choosing the lump-sum fee plan $r_{3}$, the payoff for a customer with type- $(v, q)$ is:

$$
S_{3}= \begin{cases}v q-\gamma, & q \leq q_{t}, \\ v q-\gamma-\beta p\left(q-q_{t}\right), & q>q_{t} .\end{cases}
$$

where $\gamma$ represents the lump-sum fees when the customer consumes less than $q_{t}$ amount of electricity. For the overage electricity, the unit price is $\beta p$. Thus, the same as in $\overline{S_{2}}$, the expect payoff for a customer selecting Plan 3 is

$$
\begin{aligned}
\overline{S_{3}} & =\int_{0}^{\bar{q}} S_{3} f(q) d q \\
& =\int_{0}^{q_{t}}[v q-\gamma] f(q) d q \\
& +\int_{q_{t}}^{\bar{q}}\left[v q-\gamma-\beta p\left(q-q_{t}\right)\right] f(q) d q .
\end{aligned}
$$

\section{Retail Electric Provider Profit}

The REP's profits for all plans are listed as follows.

1) Plan 1: For a type $(v, q)$ customer who chooses plan $r_{1}$, the REP's profit $\Pi_{1}$ is the payment from customers minus the electricity cost,

$$
\Pi_{1}=p q-q c,
$$

where $p$ is the market competitive price which is assumed as a constant here, and $c$ is the unit electricity cost. The REP's expected profit from Plan 1 is

$$
\bar{\Pi}_{1}=\int_{0}^{\bar{v}} \int_{0}^{\bar{q}}[p q-q c] f(q) f(v) d q d v,
$$

where $f(v)=\frac{1}{\bar{v}}$ is the PDF of customer's evaluation.

2) Plan 2: Facing a type $(v, q)$ customer who chooses plan $r_{2}$, the profit of the REP is

$$
\Pi_{2}(\alpha)=\left\{\begin{array}{lr}
\alpha p q-q c, & q<q_{t}, \\
\alpha p q_{2}+\beta p\left(q-q_{t}\right)-q c, & q_{t}<q \leq \bar{q} .
\end{array}\right.
$$

The REP's expected profit from Plan 2 is

$$
\begin{aligned}
\bar{\Pi}_{2} & =\int_{0}^{\bar{v}}\left[\int_{0}^{\overline{q_{t}}}(\alpha p q-q c) f(q) d q\right. \\
& \left.+\int_{\overline{q_{t}}}^{\bar{q}}\left(\alpha p q_{2}+\beta p\left(q-q_{t}\right)-q c\right) f(q) d q\right] f(v) d v .
\end{aligned}
$$

3) Plan 3: Facing a type $(v, q)$ customer who chooses plan $r_{3}$, the profit of the REP is

$$
\Pi_{3}(\gamma)=\left\{\begin{array}{lr}
\gamma-q c, & q \leq q_{t}, \\
\gamma+\beta p\left(q-q_{t}\right)-q c, & q_{t}<q \leq \bar{q} .
\end{array}\right.
$$

The REP's expected profit from Plan 3 is

$$
\begin{aligned}
\bar{\Pi}_{3} & =\int_{0}^{\bar{v}}\left[\int_{0}^{\overline{q_{t}}}(\gamma-q c) f(q) d q\right. \\
& \left.+\int_{\overline{q_{t}}}^{\bar{q}}\left(\gamma+\beta p\left(q-q_{t}\right)-q c\right) f(q) d q\right] f(v) d v .
\end{aligned}
$$

\section{BACKWARD InduCtion OF THE ThreE-Stage STACKELBERG GAME}

In this section, we will derive the REP's optimal decisions by backward induction. The customers' optimal decisions will be obtained firstly in Stage III. Then the REP's optimal pricing strategy will be provided in Stage II based on customers' decisions. Given REP's optimal profit under different scenarios, its optimal decision in Stage I can be derived.

\section{A. Customer's Decision in Stage III}

We start from the case when REP offers a combination of $\left\{r_{1}, r_{3}\right\}$. For a clear presentation of the close-form solution, we normalize the constant competitive price $p$ as 1 , which will not affect our final conclusion. Here we also assume that the electricity cost is less than the competitive price, thus $0<c<1$. Facing the two options, each customer will make selection to maximize its own payoff. Based on Eq. (2) and (4), we will show the specific regions that the customers who choose plan $r_{1}$ falls into. 
When $0<\gamma \leq \frac{1}{8}(6 \bar{q}-\beta \bar{q})$, customers who choose subscribe to plan $r_{1}$ fall into the region

$$
\mathcal{S}_{1}=\varnothing .
$$

Similarly, customers who choose subscribe to plan $r_{3}$ fall into the region

$$
\mathcal{S}_{3}=\left\{\frac{4 \gamma+\beta \bar{q}-\beta q_{t}}{2 \bar{q}+2 q_{t}} \leq v \leq \bar{v}\right\} .
$$

Customers who choose none of the plans fall into the region

$$
\mathcal{S}_{N}=\left\{0 \leq v<\frac{4 \gamma+\beta \bar{q}-\beta q_{t}}{2 \bar{q}+2 q_{t}}\right\}
$$

When $\gamma>\frac{1}{8}(6 \bar{q}-\beta \bar{q})$, customers who choose subscribe to plan $r_{1}$ fall into the region

$$
\mathcal{S}_{1}=\left\{p \leq v \leq \frac{4 \gamma \bar{q}-\bar{q}^{2}+\beta \bar{q}^{2}-4 \gamma q_{t}-2 \beta \bar{q} q_{t}+\beta q_{t}^{2}}{\bar{q}^{2}-2 q_{t}^{2}}\right\} .
$$

Similarly, customers who choose to order from plan $r_{3}$ fall into the region

$$
\mathcal{S}_{3}=\left\{\frac{4 \gamma \bar{q}-\bar{q}^{2}+\beta \bar{q}^{2}-4 \gamma q_{t}-2 \beta \bar{q} q_{t}+\beta q_{t}^{2}}{\bar{q}^{2}-2 q_{t}^{2}} \leq \bar{v}\right\} .
$$

Customers who choose none of the plans fall into the region

$$
\mathcal{S}_{N}=\{0 \leq v<p\} .
$$

Based on the above discussion, we are able to know customers' selection $\left(\mathcal{S}^{1}, \mathcal{S}^{3}, \mathcal{S}^{N}\right)$ are characterized by the lines $v_{1}, v_{2}$, and $v=p=1$ as illustrated in Fig. 1, where

$$
\begin{aligned}
v_{1} & =\frac{4 \gamma+\beta \bar{q}-\beta q_{t}}{2 \bar{q}+2 q_{t}}, \\
v_{2} & =\frac{4 \gamma \bar{q}-\bar{q}^{2}+\beta \bar{q}^{2}-4 \gamma q_{t}-2 \beta \bar{q} q_{t}+\beta q_{t}^{2}}{\bar{q}^{2}-2 q_{t}^{2}} .
\end{aligned}
$$

Proposition 1. When $0<\gamma \leq \frac{1}{8}(6 \bar{q}-\beta \bar{q})$, a type- $(v, q)$ customer would choose:

- Plan 1: No customer will select;

- Plan 3: if $v_{1} \leq v<\bar{v}$;

- None: if $0<v<v_{1}$.

When $\gamma>\frac{1}{8}(6 \bar{q}-\beta \bar{q})$, a type- $(v, q)$ customer would choose:

- Plan 1: if $p \leq v<v_{2}$;

- Plan 3: if $v_{2} \leq v<\bar{v}$;

- None: if $0<v<p$.

Proposition 1 shows that, when the lump-sum fee in Plan 3 is small, no customer will choose Plan 1. There are customers select Plan 1 only when lump-sum fee is larger than a certain value. Furthermore, Plan 1 will only be selected by customers with low evaluation, who are likely to consume less electricity. While customers with high evaluation will be less sensitive to price, they would prefer Plan 3. As for the combination of $\left\{r_{1}, r_{2}\right\}$, the formulation and analysis are similar to that of the combination of $\left\{r_{1}, r_{3}\right\}$, and they can be obtained following the same procedure.

\section{B. Optimal Pricing in Stage II}

As the electricity supplier, REP's objective is to maximize the profit while providing satisfactory services to the electricity customers. In stage II, the REP will determine the optimal pricing strategy based on customers' decisions in Stage III. Since the price in Plan 1 is a constant, we first derive the optimal prices in Plan 2 and 3 when only one of the 3 plans are offered, then derive the optimal pricing strategy when the REP offering the combination of $\left\{r_{1}, r_{3}\right\}$.

1) Plan 2: Based on the definition of REP's expected profit in Eq. (7), we derive the REP's optimal pricing strategy for Plan 2 in Proposition 2.

Proposition 2. The REP's optimal pricing in Plan 2 is

$$
\alpha^{*}=\frac{-2 \beta \bar{q}^{2}+c \bar{q}^{2}+4 \beta \bar{q} q_{t}-2 \beta q_{t}^{2}+\bar{q}^{2} \bar{v}}{2\left(2 \bar{q}-q_{t}\right) q_{t}} .
$$

2) Plan 3: Based on the definition of REP's expected profit in Eq. (8), we derive the REP's optimal pricing strategy for Plan 3 in Proposition 3.

Proposition 3. The REP's optimal pricing in Plan 3 is

$$
\gamma^{*}=\frac{-3 \beta \bar{q}^{2}+2 c \bar{q}^{2}+5 \beta \bar{q} q_{t}-2 \beta q_{t}^{2}+2 \bar{q}^{2} \bar{v}+2 \bar{q} q_{t} \bar{v}}{8 \bar{q}} .
$$

3) Combination $\left\{r_{1}, r_{3}\right\}$ : When both Plan 1 and Plan 3 are both available at the same time, the REP has 3 choices: offing 2 plans together, Plan 1 only, or Plan 3 only. Based on customers' decision obtained from the previous subsection, and the REP's payoff functions defined in Eqs. (5) and (8), we show the REP's optimal pricing strategy in Proposition 4.

Proposition 4. When $0<\gamma \leq \frac{1}{8}(6 \bar{q}-\beta \bar{q})$, the REP offers Plan 3 only, the optimal pricing is the same as in Proposition 3.

When $\gamma>\frac{1}{8}(6 \bar{q}-\beta \bar{q})$, REP offers Plan 1 and 3 at the same time ready for customers to choose, the optimal pricing is

$\gamma^{*}=\frac{2(-1+4 \beta) \bar{q}^{2} q_{t}+2 \beta q_{t}^{3}+\bar{q}^{3}(3-3 \beta+\bar{v})-\bar{q} q_{t}^{2}(7 \beta+2 \bar{v})}{8 \bar{q}\left(\bar{q}-q_{t}\right)}$.

\section{Optimal Decision in Stage I}

Under the optimal prices obtained from the previous section, we first derive the optimal profit in Plan 1, 2, and 3 when only one of the 3 plans are offered, then derive the optimal profit when the REP offering the combination of $\left\{r_{1}, r_{3}\right\}$.

1) Plan 1: Based on the definition of REP's expected profit in Eq. (5), we derive the optimal profit when REP only offers Plan 1.

Proposition 5. Under the market competitive price, the REP's optimal profit is

$$
\Pi_{1}^{*}=\frac{1}{2}(\bar{q}-c)
$$




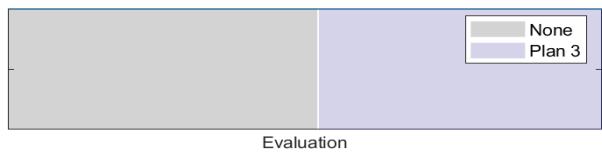

(a) $0<\gamma \leq \frac{1}{8}(6 \bar{q}-\beta \bar{q})$.

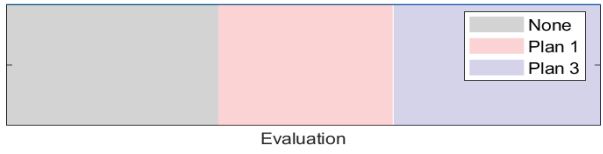

(b) $\gamma>\frac{1}{8}(6 \bar{q}-\beta \bar{q})$.

Fig. 1: The customer's optimal decisions.

2) Plan 2: Based on the definition of REP's expected profit in Eq. 7, we derive the optimal profit when REP only offers Plan 2.

Proposition 6. Under the market competitive price, the REP's optimal profit is

$$
\Pi_{2}^{*}=\frac{\bar{q}(c-\bar{v})^{2}}{8 \bar{v}} .
$$

3) Plan 3: Based on the definition of REP's expected profit in Eq. (8), we derive the optimal profit when REP only offers Plan 3.

Proposition 7. Under the market competitive price, the REP's optimal profit is

$$
\Pi_{3}^{*}=\frac{\left(\beta\left(\bar{q}^{2}-3 \bar{q} q_{t}+2 q_{t}^{2}\right)+2 \bar{q}\left(-c \bar{q}+\left(\bar{q}+q_{t}\right) \bar{v}\right)\right)^{2}}{32 \bar{q}^{2}\left(\bar{q}+q_{t}\right) \bar{v}} .
$$

4) Combination $\left\{r_{1}, r_{3}\right\}$ : Based on the definition of REP's expected profit in Eq. (4), the optimal profit of the REP can achieve when offering Plan 1 and 3 at the same time.

Proposition 8. When $0<\gamma \leq \frac{1}{8}(6 \bar{q}-\beta \bar{q})$, the REP offers Plan 3 only, the optimal profit is the same as in Proposition 7.

When $\gamma>\frac{1}{8}(6 \bar{q}-\beta \bar{q})$, the REP offers Plan 1 and 3 together, the optimal profit can also get a close form solution.

The close-form solution here has a long equation which we will provide in our technical report.

Given the REP's optimal profits under different scenarios, we can conduct a comparison between serving Plan 3 only or the two plans together. Then we will be able to choose the one that maximizes REP's profit. The REP's decision can be summarized in the following proposition.

Proposition 9. When $0<\bar{v} \leq \frac{1}{3}(3-\sqrt{3})$,

- $0 \leq c<1$, offering Plan 3 only.

When $\frac{1}{3}(3-\sqrt{3})<\bar{v} \leq \frac{1}{3}(3+\sqrt{3})$,

- $0 \leq c \leq-\frac{3}{2}(-2+\bar{v})-\frac{1}{2} \sqrt{3} \sqrt{4-4 \bar{v}+\bar{v}^{2}}$, offering Plan 3 only;

- $-\frac{3}{2}(-2+\bar{v})-\frac{1}{2} \sqrt{3} \sqrt{4-4 \bar{v}+\bar{v}^{2}} \leq c \leq 1$, offering Plan 1 and 3 together.

When $\frac{1}{3}(3+\sqrt{3})<\bar{v}<2$,

- $0 \leq c \leq-\frac{3}{2}(-2+\bar{v})-\frac{1}{2} \sqrt{3} \sqrt{4-4 \bar{v}+\bar{v}^{2}}$, offering Plan 3 only;

- $-(3 / 2)(-2+\bar{v})-\frac{1}{2} \sqrt{3} \sqrt{4-4 \bar{v}+\bar{v}^{2}} \leq c \leq-\frac{3}{2}(-2+$ $\bar{v})+\frac{1}{2} \sqrt{3} \sqrt{4-4 \bar{v}+\bar{v}^{2}}$, offering Plan 1 and 3 together;

- $-\frac{3}{2}(-2+\bar{v})+\frac{1}{2} \sqrt{3} \sqrt{4-4 \bar{v}+\bar{v}^{2}} \leq c \leq 1$, offering Plan 3 only.
When $\bar{v} \geq 2$,

- $0 \leq c<1$, offering Plan 3 only.

\section{Simulation Results and AnAlysis}

In this section, numerical simulations will be given to illustrate how REP's profit is impacted by the key parameters such as customer's evaluation, electricity consumption, and REP's cost on electricity in Fig. (2). Furthermore, REP's optimal decisions under the combination of $\left\{r_{1}, r_{3}\right\}$ are also provided in Fig. 3.

\section{A. Comparisons Among Plans}

From Fig. 2 we see that the REP's profit is the highest when offering Plan 3, then Plan 2. The flat-rate Plan 1 brings REP the lowest profit. This result actually coincides with our observations in daily life. The flat-rate Plan 1 is the old way for charging customers, which are gradually replaced by the multi-stage pricing strategy in recent decades, which is a general case of our proposed Plan 2, when there are multiple stages instead of 2 . For the lump-sum fee plan, it has been offered by many REPs such as Reliant and Direct Energy in North America, and is one of the most popular electricity plans among residential customers. The lump-sum plan encounrages users to consume more electricity, and charges customer a subscription fee no matter if they use up the electricity or not. Thus many customers encounter the sunken cost which turns into the REP's profit.

Fig. 2a shows that REP's profit is an increasing function of customer's evaluation, which is quite intuitive. Since the more customers evaluate the electricity, the higher price they would like to pay, which directly increases REP's income when the electricity cost is fixed. For the flat-rate Plan 1 since we normalize it as a constant, thus the REP's profit is independent of customer's evaluation. Fig. $2 b$ shows that REP's profit is a decreasing function of electricity cost, which is also an intuitive result. Since the higher the unit price that the REP buys the electricity from power plant, the higher price customers will be charged. In return, facing high price, less customers will choose the plan, results in less profit for REP. Fig. 2c shows that REP's profit is an increasing function of customer consumption. Since the more electricity the customers consumes, the more they need to pay to the REP.

\section{B. Retail Electric Provider's Optimal Decision}

In Fig. 3, the optimal choices for maximizing REP's profit are provided under different settings. Fig. 3 shows REP is capable of offering both Plan 1 and 3 together at the middle 


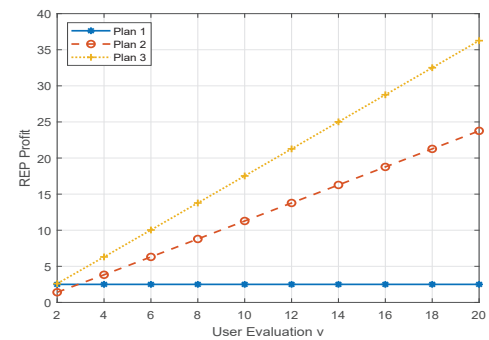

(a) Evaluation.

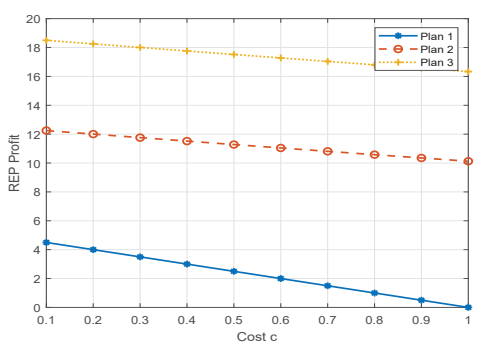

(b) Cost.

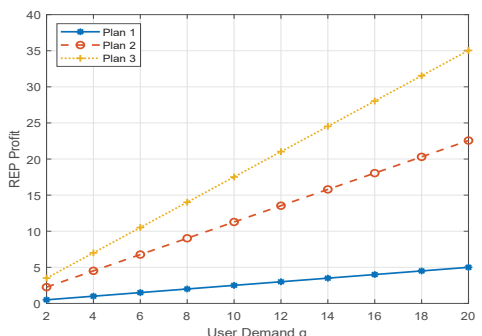

(c) Demand.

Fig. 2: The service provider's optimal profit under different system setting.

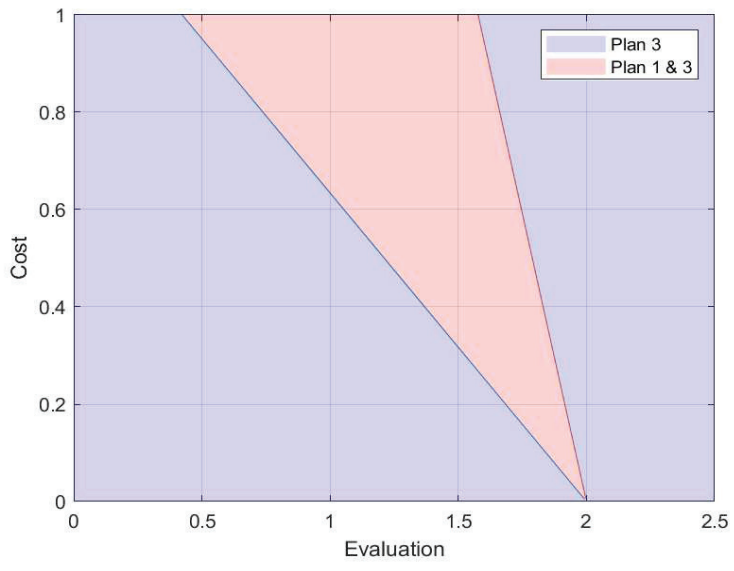

Fig. 3: The REP's optimal decision.

red region, but need to switch to offer Plan 3 only in the blue region. This result shows that when customer's evaluation is low, Plan 1 is possible to bring competitive profit compared to Plan 3. But as customer's evaluation increases, the REP should simply serve Plan 3 since it brings much higher profit than Plan 1. The similar conclusion can be also found out in Fig. 2.

\section{CONCLUSIONS}

In this paper, we have studied REP's optimal pricing strategy when providing different electricity plans for residential customers' selections. First, we derive customers' optimal decisions by considering the pricing strategy in different plans. Then, we provide the REP's optimal pricing strategies for each plan. In the end, we give the optimal decisions for REP on the specific plan(s) to serve when offering Plan 1 and 3 at the same time. Through analysis, we find out that Plan 1 is optimal only when customers have low evaluation, while customers with high evaluation and high demand would prefer Plan 3. Furthermore, when the lump-sum fee in Plan 3 is small, no customer will choose Plan 1.

Our work opens several directions in the future. First, we assume the REP's electricity cost is a constant value, but in reality REP's electricity also encounters an increase when the purchased amount electricity is overage. Thus, it is necessary to model REP's cost in a more general way. Second, REP usually buys electricity from power plant with a lump-sum fee and there will be additional cost if electricity are not consumed, which is similar to customer's Plan 3. Less or over consumptions both deteriorates REP's profit. For future research, it is also meaningful to maximize REP's profit while considering the stability of the smart grid system.

\section{REFERENCES}

[1] A. Khalid, N. Javaid, M. Guizani, M. Alhussein, K. Aurangzeb, and M. Ilahi, "Towards dynamic coordination among home appliances using multi-objective energy optimization for demand side management in smart buildings," Ieee Access, vol. 6, pp. 19509-19529, 2018.

[2] J. Lin, W. Yu, and X. Yang, "Towards multistep electricity prices in smart grid electricity markets," IEEE Transactions on Parallel and Distributed Systems, vol. 27, no. 1, pp. 286-302, 2016.

[3] Srp time-of-use price plan. [Online]. Available: https://www.srpnet.com/prices/home/tou.aspx

[4] L. Gelazanskas and K. A. Gamage, "Demand side management in smart grid: A review and proposals for future direction," Sustainable Cities and Society, vol. 11, pp. 22-30, 2014.

[5] A. Cooper and K. Schefter. Electric vehicle sales forecast and the charging infrastructure required through 2030. [Online]. Available: http://www.edisonfoundation.net/iei/publications/Documents/

[6] Y. Zhou, N. Maxemchuk, X. Qian, and C. Wang, "The fair distribution of power to electric vehicles: An alternative to pricing," in 2014 IEEE International Conference on Smart Grid Communications (SmartGridComm). IEEE, 2014, pp. 686-691.

[7] J. Ma, H. H. Chen, L. Song, and Y. Li, "Residential load scheduling in smart grid: A cost efficiency perspective," IEEE transactions on smart grid, vol. 7, no. 2, pp. 771-784, 2016.

[8] Q. Huang, X. Li, J. Zhao, D. Wu, and X.-Y. Li, "Social networking reduces peak power consumption in smart grid," IEEE Transactions on Smart Grid, vol. 6, no. 3, pp. 1403-1413, 2015.

[9] D. Srinivasan, S. Rajgarhia, B. M. Radhakrishnan, A. Sharma, and H. Khincha, "Game-theory based dynamic pricing strategies for demand side management in smart grids," Energy, vol. 126, pp. 132-143, 2017.

[10] Z. Wang and R. Paranjape, "Optimal residential demand response for multiple heterogeneous homes with real-time price prediction in a multiagent framework," IEEE transactions on smart grid, vol. 8, no. 3, pp. 1173-1184, 2017.

[11] K. Zhou and S. Yang, "Demand side management in china: The context of china's power industry reform," Renewable and Sustainable Energy Reviews, vol. 47, pp. 954-965, 2015.

[12] N. Yu, L. Tesfatsion, and C.-C. Liu, "Financial bilateral contract negotiation in wholesale electricity markets using nash bargaining theory," IEEE Transactions on Power Systems, vol. 27, no. 1, pp. 251-267, 2012.

[13] X. Lei, D.-x. Yu, and X.-l. Bai, "Research on multistep electricity price model with bidirectional regulation for large consumers," in 2010 International Conference on Electrical and Control Engineering, 2010, pp. 4114-4117.

[14] M. Muratori and G. Rizzoni, "Residential demand response: Dynamic energy management and time-varying electricity pricing," IEEE Transactions on Power systems, vol. 31, no. 2, pp. 1108-1117, 2016. 